\title{
The 1-Laplacian, the $\infty$-Laplacian and Differential Games
}

\author{
Lawrence C. Evans
}

\begin{abstract}
This paper considers the $p$-Laplacian PDE for $p=1, \infty$ and some interesting new game theoretic interpretations, due to Kohn-Serfaty [K-S] and to Peres-Schramm-Sheffield-Wilson [P-S-S-W].
\end{abstract}

\section{Introduction}

This informal, expository paper discusses the degenerate/singular diffusion PDE

$$
\operatorname{div}\left(|D u|^{p-2} D u\right)=0
$$

in the extreme limiting cases $p=1, p \rightarrow \infty$, and explains also some interesting game theoretic interpretations due to Kohn-Serfaty [K-S] and to Peres-SchrammSheffield-Wilson [P-S-S-W]. I additionally lace the exposition with various general comments, taking this occasion to preach a bit about some guiding principles of nonlinear analysis and nonlinear PDE.

This is a recapitulation and follow-up to my lecture in Paris during the summer of 2004, at the conference in honor of Haim Brezis. It is a real pleasure to contribute a paper to this volume: Haim has long been one of my mathematical heros, starting with my reading as a graduate student of his monograph Operateurs Maximaux Monotones et Semi-groupes de Contractions dans les Espaces de Hilbert and continuing to this day. Of his countless contributions to our field, I would particularly like to single out Haim's service as editor of the major Birkhäuser series Progress in Nonlinear Differential Equations and Their Applications.

\section{Degenerate diffusions, extreme cases}

2.1 Overview. It seems to me that within nonlinear analysis we should always be looking for unifying, guiding principles that cut across the boundaries of specific problems. And it is by studying in detail appropriately simple models that we often stumble upon general principles.

The p-Laplacian for $1<p<\infty$. A greatly studied such model problem is the nonlinear PDE

$$
\Delta_{p} u=0
$$

1991 Mathematics Subject Classification. 35J15, 91A20.

Key words and phrases. Minimax, nonlinear diffusion PDE, differential games.

Supported in part by NSF grant DMS-0500452. 
holding in some open set $U \subseteq \mathbb{R}^{n}$, where we write

$$
\Delta_{p} u:=\operatorname{div}\left(|D u|^{p-2} D u\right)
$$

for the $p$-Laplacian. This is of course the Euler-Lagrange equation for minimizing the functional

$$
I_{p}[v]:=\frac{1}{p} \int_{U}|D v|^{p} d x
$$

subject to given, but here unspecified, boundary conditions.

Our quasilinear PDE is elliptic, but is degenerate wherever $D u=0$ for $2<$ $p<\infty$ and is singular wherever $D u=0$ for $1<p<2$. It is easy to show there exists a minimizer $u=u_{p}$ belonging to the Sobolev space $W^{1, p}$, but examples show $u \notin C^{2}$ in general. When $2<p<\infty, \mathrm{K}$. Uhlenbeck and N. Ural'ceva independently showed that $u \in C^{1, \alpha}$ for some $\alpha=\alpha(p)$; and years later I gave another proof in [E1]. The idea of these proofs is roughly as follows: On the set $\{|D u|>0\}$ the PDE is nondegenerate and the usual strong elliptic estimates apply. On $\{|D u|=0\}$ the PDE degenerates, but there we know that in fact the gradient $D u=0$. (This insight is far less useful for a PDE that degenerates also for other values of $D u$.)

2.2 Extreme cases. One important principle of mathematics is that extreme cases reveal interesting structure. We illustrate this bit of mathematical faith by putting $p=1, p \rightarrow \infty$ in (2.1).

The 1-Laplacian. Setting $p=1$ in (2.1) is easy and gives us the PDE

$$
\Delta_{1} u:=\operatorname{div}\left(\frac{D u}{|D u|}\right)=0
$$

assuming of course $u$ is smooth and $|D u| \neq 0$. The nonlinear operator $\Delta_{1}$ is called the 1-Laplacian. For later reference, we rewrite into nondivergence form:

$$
\Delta_{1} u:=\frac{1}{|D u|}\left(\delta_{i j}-\frac{u_{x_{i}} u_{x_{j}}}{|D u|^{2}}\right) u_{x_{i} x_{j}}=0 .
$$

Since the vector

$$
\nu:=\frac{D u}{|D u|}
$$

is perpendicular to each level set of $u$, we see that our 1-Laplacian PDE (2.3) describes "isotropic diffusion within each level surface, with no diffusion across different level surfaces." Of course in general a solution of a PDE like (2.4) must be interpreted in some suitable weak sense, in this case as a viscosity solution, as for instance in $[\mathbf{E}-\mathbf{S p}]$.

The $\infty$-Laplacian. Sending $p \rightarrow \infty$ is trickier. For this, first rewrite $(2.1)$ into nondivergence form:

$$
|D u|^{p-2} \Delta u+(p-2)|D u|^{p-4} u_{x_{i}} u_{x_{j}} u_{x_{i} x_{j}}=0 .
$$

Divide by $|D u|^{p-2}(p-2)$ and send $p \rightarrow \infty$ :

$$
\Delta_{\infty} u:=\frac{u_{x_{i}} u_{x_{j}}}{|D u|^{2}} u_{x_{i} x_{j}}=0 .
$$

The nonlinear operator $\Delta_{\infty}$ is called the $\infty$-Laplacian. Observe that the $\infty$ Laplacian PDE (2.5) formally describes "diffusion across different level surfaces, with no diffusion within each level surface." This interpretation is somehow "dual" 
to that for the 1-Laplacian. Again, a solution of (2.5) must rigorously be interpreted as a viscosity solution: see Aronsson-Crandall- Juutinen $[\mathbf{A - C - J}]$ for details.

The next sections review some of the literature on the PDE (2.3), (2.5).

\section{More interpretations of the 1- and $\infty$-Laplacians}

3.1 The 1-Laplacian. Look now at the boundary-value problem

$$
\left\{\begin{aligned}
\Delta_{1} u=0 & \text { in } U \\
u=g & \text { in } \partial U
\end{aligned}\right.
$$

for a given function $g: \partial U \rightarrow \mathbb{R}$.

Variational interpretation. This nonlinear boundary value problem formally corresponds the variational problem of minimizing

$$
I_{1}[v]=\int_{U}|D v| d x
$$

subject to the boundary condition that $v=g$ on $\partial U$.

Geometric interpretation. Now let

$$
\Gamma_{c}:=\{x \in c \mid u(x)=c\}
$$

denote a level set of $u$, which we suppose is a smooth hypersurface, with unit normal $\boldsymbol{\nu}=\frac{D u}{|D u|}$. The mean curvature of $\Gamma_{c}$ is

$$
H=\operatorname{div}(\boldsymbol{\nu})=\operatorname{div}\left(\frac{D u}{|D u|}\right) .
$$

So our PDE $\Delta_{1} u=0$ asserts that "each level surface of $u$ has mean curvature zero".

Estimates and regularity. The foregoing geometry involves each level set $\Gamma_{c}$ of $u$, and is consequently unchanged if we relabel the level sets. This means that our PDE is invariant under an arbitrary change of dependent variable:

$$
\text { If } u \text { solves } \Delta_{1} u=0 \text { and } w=\phi(u) \text {, then } \Delta_{1} w=0 \text { also. }
$$

But this invariance is bad for analysis, since it implies that there can be no interior a priori estimate on a modulus of continuity of $u$ or its gradient $D u$.

We discuss next similar insights for the $\infty$-Laplacian.

3.2 The $\infty$-Laplacian. As a kind of dual problem to (3.1) consider now the boundary value problem

$$
\left\{\begin{aligned}
\Delta_{\infty} u=0 & \text { in } U \\
u=g & \text { on } \partial U .
\end{aligned}\right.
$$

Variational interpretation. Assume that $g: \partial U \rightarrow \mathbb{R}$ is Lipschitz continuous. G. Aronsson $[\mathbf{A} \mathbf{1}]$ has proposed the " $L$ variational problem" of finding an extension $u$ such that

$$
\left\{\begin{array}{l}
\text { for each open set } V \subseteq U \\
\quad \operatorname{ess-sup}_{V}|D u| \leq \operatorname{ess-sup}_{V}|D v| \\
\text { for all } v=u \text { on } \partial V .
\end{array}\right.
$$

It turns out that requiring $u$ to satisfy (3.4) is equivalent to asking $u$ to be a viscosity solution of (3.3). The really basic problem concerns the uniqueness of 
functions satisfying (3.4), which Bob Jensen proved in his important paper $[\mathbf{J}]$. See Aronsson-Crandall-Juutinen $[\mathbf{A}-\mathbf{C}-\mathbf{J}]$ for more.

Geometric interpretation. I am unaware of any geometric interpretation of (3.3) and pose this as an open question. Consider the ODE

$$
\dot{\mathbf{x}}=\boldsymbol{\nu}(\mathbf{x})=\frac{D u}{|D u|}(\mathbf{x}),
$$

with flow lines normal to the level surface of $u$. Is there any purely geometric way to explain the corresponding trajectories? Whatever answer the reader comes up with should in particular explain the flow for Aronsson's solution (3.5), mentioned later.

Estimates and regularity. Regularity issues for the $\infty$-Laplacian are very hard. O. Savin $[\mathbf{S}]$ has recently made a major breakthrough, proving that if $n=2$ and $u$ is the viscosity solution of (3.3), then

$$
u \in C^{1}(U) .
$$

It remains an outstanding problem to prove $C^{1}$-regularity in dimensions $n \geq 3$.

The Harnack inequality states that for each $V \subset \subset U$ there exists $C=C(V)$ such that if $u \geq 0$, then

$$
\max _{V} u \leq C \min _{V} u
$$

I proved in [E3] that if $u$ is smooth, then $|D u|$ also satisfies a Harnack inequality:

$$
\max _{V}|D u| \leq C \min _{V}|D u|
$$

and this has the remarkable consequence that a smooth, nonconstant solution of (3.3) can have no critical point within $U$. Aronsson had earlier proved this for $n=2$ dimensions. However, this conclusion is dead false for general weak (that is, viscosity) solutions, a fundamental example of which for $n=2$ is

$$
u=x_{1}^{\frac{4}{3}}-x_{2}^{\frac{4}{3}},
$$

which Aronsson found by separating variables.

Blow-up limits. Crandall, Gariepy and I have shown in $[\mathbf{C - E}-\mathbf{G}]$ and $[\mathbf{C - E}]$ that for each point $x_{0} \in U$ that

$$
\left\{\begin{array}{l}
\text { if the limit } v(x):=\lim _{r_{j} \rightarrow 0} \frac{u\left(x_{0}+r_{j} x\right)-u\left(x_{0}\right)}{r_{j}} \text { exists, } \\
\text { then } v(x)=\langle a, x\rangle \text { is linear. }
\end{array}\right.
$$

This is an interesting assertion in view of the basic open question of regularity for weak solutions. Note carefully that (3.6) does not assert $u$ to be differentiable at the point $x_{0}$ since different rescaled, blow-up sequences could possibly converge to different linear functions. 


\section{Game theory interpretations}

4.1 Minimax formulas. I again indulge myself in this section with a bit of philosophy concerning nonlinear problems.

Convexity. First, let me review convexity. A key point is that convexity is a kind of "one-sided" linearity. More precisely, if $\Phi: \mathbb{R}^{n} \rightarrow \mathbb{R}$ is convex, we can write

$$
\Phi(x)=\max _{\alpha}\left\{\left\langle a^{\alpha}, x\right\rangle+b^{\alpha}\right\}
$$

for appropriate $a^{\alpha} \in \mathbb{R}^{n}, b^{\alpha} \in \mathbb{R}$. This representation formula suggests why convexity is a fundamental hypothesis for many nonlinear problems: linear and affine functions are simple, and "max" is a simple operation. Consequently, convex functions are (relatively) simple to study.

General nonlinearities. Suppose now $\Psi: \mathbb{R}^{n} \rightarrow \mathbb{R}$ is an arbitrary, say Lipschitz continuous, function. Then we can write

$$
\Psi(x)=\min _{\beta} c_{\beta}(x),
$$

where each function $c_{\beta}(\cdot)$ is convex. For example, we can touch each point of the graph of $u$ from above by a round cone. But as above we have

$$
c_{\beta}(x)=\max _{\alpha}\left\{\left\langle a^{\alpha, \beta}, x\right\rangle+b^{\alpha, \beta}\right\}
$$

for appropriate $a^{\alpha, \beta} \in \mathbb{R}^{n}$ and $b^{\alpha, \beta} \in \mathbb{R}$; and hence

$$
\Psi(x)=\min _{\beta} \max _{\alpha}\left\{\left\langle a^{\alpha, \beta}, x\right\rangle+b^{\alpha, \beta}\right\} .
$$

This formula shows that an essentially arbitrary nonlinearity can be written as a maximin of affine mappings. I found a more explicit formula in [E2]:

$$
\Psi(x)=\max _{\alpha \in \mathbb{R}^{n}} \min _{\beta \in \mathbb{R}^{n}}\left\{\int_{0}^{1}\langle D \Psi((1-\lambda) \alpha+\lambda \beta), x-\alpha\rangle d \lambda+\Psi(\alpha)\right\} ;
$$

and it turns out that "minimax = maximin" for this particular representation.

With these formulas in mind, we can extend the heuristics above. Linear and affine functions are simple, "max" is a simple operation and "min" is a simple operation. Consequently, since essentially any nice function admits the maximin representations (4.2) or (4.3), we in principle have a general tool for nonlinear analysis.

Control theory and game theory. There is another way to view these comments. For convex problems, with max-type structure, we expect to have optimization or control theory representation formulas for solutions. And likewise for wide classes of problems with minimax- and/or maximin-type structure, we can expect to find game theoretic interpretations. (See for instance $[\mathbf{E}-\mathbf{S o}]$.)

In the next sections, we illustrate this principle for the 1- and $\infty$-Laplacians.

4.2 A random "tug-of-war" game. Following Peres-Schramm-SheffieldWilson [P-S-S-W] let us introduce for each $\epsilon>0$ a random two-person, zero-sum game, in which Player $I$ wants to minimize the expected payoff and player $I I$ wants to maximize the expected payoff.

The rules of play are:

Step 1: At each time $t_{k}=k \epsilon$, players $I$ and $I I$ flip a fair coin. 
Step 2: The winner selects a unit vector $v \in \mathbb{R}^{n}$ and moves from the current position $x_{k}$ to the new position

$$
x_{k+1}:=x_{k}+\epsilon v .
$$

Step 3: The game ends when our process first exits $U$, that is, when $x^{*}=x_{k+1} \notin U$. The payoff is then $g\left(x^{*}\right)$, for a given function $g: \partial U \rightarrow \mathbb{R}$.

Dynamic programming and PDE. The method of dynamic programming lets us cast this problem into analytic terms. Let $u^{\epsilon}(x)$ denote expected payoff, given we start at $x=x_{0}$. Then

$$
u^{\epsilon}(x)=\frac{1}{2}\left(\max _{\partial B(x, \epsilon)} u^{\epsilon}+\min _{\partial B(x, \epsilon)} u^{\epsilon}\right) .
$$

The idea is that if player I wins the coin flip, he moves the point on $\partial B(x, \epsilon)$ where $u^{\epsilon}$ is smallest; whereas if player II wins the coin flip, she moves the point on $\partial B(x, \epsilon)$ where $u^{\epsilon}$ is largest. These events occur with equal probability $1 / 2$. (This functional identity (4.4) had been previously noted by Oberman $[\mathbf{O}]$ and Le Gruyer $[\mathbf{L G}]$.)

It is not so hard to guess from (4.4) that if $u^{\epsilon} \rightarrow u$ as $\epsilon \rightarrow 0$, then $u$ should solve the boundary-value problem

$$
\left\{\begin{aligned}
\Delta_{\infty} u=0 & \text { in } U \\
u=g & \text { on } \partial U .
\end{aligned}\right.
$$

To see this, note that if $D u^{\epsilon}(x) \neq 0$, then

$$
\begin{aligned}
& \max _{\partial B(x, \epsilon)} u^{\epsilon} \approx u^{\epsilon}\left(x+\epsilon D u^{\epsilon}(x) /\left|D u^{\epsilon}(x)\right|\right) \\
& \min _{\partial B(x, \epsilon)} u^{\epsilon} \approx u^{\epsilon}\left(x-\epsilon D u^{\epsilon}(x) /\left|D u^{\epsilon}(x)\right|\right) .
\end{aligned}
$$

Hence the functional equation (4.4) implies

$$
u^{\epsilon}\left(x+\epsilon D u^{\epsilon}(x) /\left|D u^{\epsilon}(x)\right|\right)+u^{\epsilon}\left(x-\epsilon D u^{\epsilon}(x) /\left|D u^{\epsilon}(x)\right|\right)-2 u^{\epsilon}(x) \approx 0 .
$$

Divide by $\epsilon^{2}$ and send $\epsilon \rightarrow 0$, to obtain (4.5).

This very rough derivation is suspicious, since we in fact do not know the functions $u^{\epsilon}$ to be smooth, nor that the error term in (4.6) is of order $o\left(\epsilon^{2}\right)$. Nevertheless the conclusion is correct, provided we understand $u$ as a viscosity solution: see Peres-Schramm-Sheffield-Wilson $[\mathbf{P}-\mathbf{S}-\mathbf{S}-\mathbf{W}]$ (or $[\mathbf{B}-\mathbf{E}-\mathbf{J}]$ ) for more. And very recently Peres and Sheffield in $[\mathbf{P}-\mathbf{S}]$ have introduced a stochastic tug-of-war interpretation of the $p$-Laplacian operator for $1<p<\infty$.

4.3 A deterministic "pusher-chooser" game. Kohn and Serfaty [K-S] have introduced a different two-person, zero-sum game, but one with clear similarities. Again, player $I$ wants to minimize the expected payoff and player $I I$ wants to maximize the expected payoff.

The rules of play are now:

Step 1: At each time $t_{k}=k \epsilon^{2}$, player $I$ selects a unit vector $v \in \mathbb{R}^{2}$.

Step 2: Player $I I$ then selects $b \in\{1,-1\}$ and moves from the current position $x_{k}$ to the new position

$$
x_{k+1}:=x_{k}+\sqrt{2} \epsilon b v .
$$

Step 3: The game terminates when $x^{*}=x_{k+1} \notin U$, and the payoff is

$$
t^{*}=\text { time the process exits } U \text {. }
$$


In other words, player $I$ wants to exit from $U$ as quickly as possible, and player $I I$ wants to prevent this as long as possible.

Dynamic programming and PDE. As before, let $u^{\epsilon}(x)$ denote the payoff, given we start at $x=x_{0}$. By analogy with (4.4), we have the functional equation

$$
u^{\epsilon}(x)=\min _{|v|=1} \max _{b= \pm 1}\left\{\epsilon^{2}+u^{\epsilon}(x+\sqrt{2} \epsilon b v)\right\} .
$$

Again, the interesting question is understanding what happens as $\epsilon \rightarrow 0$. Note firstly that if $D u^{\epsilon}(x) \neq 0$, then Player I should certainly select $v= \pm D u^{\epsilon}(x)^{\perp} /\left|D u^{\epsilon}(x)\right|$, a unit vector perpendicular to $D u^{\epsilon}(x)$. Otherwise, Player II could by appropriately choosing $b= \pm 1$ force a move to a new position where $u^{\epsilon}$ is strictly larger than its value at $x$.

For definiteness, assume player I takes $v=D u^{\epsilon}(x)^{\perp} /\left|D u^{\epsilon}(x)\right|$. Then the functional equation (4.7) gives

$$
\max _{b= \pm 1}\left\{\epsilon^{2}+u^{\epsilon}\left(x+\sqrt{2} \epsilon b D u^{\epsilon}(x)^{\perp} /\left|D u^{\epsilon}(x)\right|\right)\right\}-u^{\epsilon}(x)=0
$$

and then our expanding $u^{\epsilon}$ in a Taylor series about $x$ shows

$$
\max _{b= \pm 1}\left\{\epsilon^{2}+\frac{b^{2}}{\left|D u^{\epsilon}(x)\right|^{2}}\left\langle D u^{\epsilon}(x)^{\perp}, D^{2} u^{\epsilon}(x) D u^{\epsilon}(x)^{\perp}\right\rangle\right\} \approx 0 .
$$

Now assume that $u^{\epsilon} \rightarrow u$. Our dividing (4.8) by $\epsilon^{2}$ and letting $\epsilon \rightarrow 0$ yields the PDE

$$
-\frac{1}{|D u|^{2}}\left\langle D u^{\perp}, D^{2} u D u^{\perp}\right\rangle=1
$$

Since we are in $n=2$ dimensions, we can rewrite, to obtain

$$
\left\{\begin{aligned}
-|D u| \Delta_{1} u & =1 & & \text { in } U \\
u & =0 & & \text { on } \partial U .
\end{aligned}\right.
$$

Our formal derivation is again very suspicious; but the conclusion is valid, as proved by Kohn and Serfaty, provided we interpret $u$ as a viscosity solution.

Geometric interpretation. J. Spruck and I in [E-Sp] have previously investigated the boundary-value problem (4.9). The geometric interpretation is that the level curves of $u$ evolve by curvature motion, starting at $\partial U=\{x \mid u(x)=0\}$. Consult Kohn and Serfaty's paper $[\mathbf{K}-\mathbf{S}]$ for some pictures and more explanation as to why curvature motion is in fact relevant for their pusher-choose game.

Questions. In summary, boundary-value problem (4.9) for the pusher-chooser game entails the 1-Laplacian, whereas the corresponding problem (4.5) for the tugof-war game involves the $\infty$-Laplacian.

Is there a philosophical explanation for this? Are these problems somehow "dual"?

In view of my previous comments about minimax representations, it is perhaps not so surprising that our PDE have game theoretic interpretations, but in what sense are these particular games "natural"? 


\section{Generalizations}

Generalization: other dynamics, running costs. Barron, Jensen and I in [B-E-J] have generalized the tug-of-war problem by assuming now the dynamics to be

$$
\begin{cases}\frac{d \mathbf{x}(\tau)}{d \tau}=\mathbf{f}(\mathbf{x}(\tau), \boldsymbol{\eta}(\tau)) & \text { for player } I \\ \frac{d \mathbf{x}(\tau)}{d \tau}=-\mathbf{f}(\mathbf{x}(\tau), \boldsymbol{\zeta}(\tau)) & \text { for player } I I\end{cases}
$$

for times $0<\tau<t^{*}$. We also introduce both a terminal payoff and a running cost:

$$
P(\boldsymbol{\eta}(\cdot), \boldsymbol{\zeta}(\cdot)):=g\left(\mathbf{x}\left(T_{x}\right)\right)+\int_{0}^{t^{*}} \pm h(\mathbf{x}(\tau), \boldsymbol{\alpha}(\tau)) d \tau .
$$

Here $\boldsymbol{\alpha}(\cdot)$ denotes $\boldsymbol{\eta}(\cdot)$ when $I$ is playing and $\boldsymbol{\zeta}(\cdot)$ when $I I$ is playing. The " \pm " term means that the running payoff is $-h(\mathbf{x}(\tau), \boldsymbol{\eta}(\tau))$ when Player I controls the dynamics, and is $h(\mathbf{x}(\tau), \boldsymbol{\zeta}(\tau))$ when Player II controls the dynamics.

The dynamic programming formula analogous to (4.4) for the value function now reads

$$
\begin{aligned}
u^{\epsilon}(x)=\frac{1}{2}\left(\operatorname { m a x } _ { | y | \leq 1 } \left[u^{\epsilon}(x+\epsilon \mathbf{f}(x, y))-\right.\right. & \epsilon h(x, y)] \\
& \left.+\min _{|z| \leq 1}\left[u^{\epsilon}(x-\epsilon \mathbf{f}(x, z))+\epsilon h(x, z)\right]\right) .
\end{aligned}
$$

We make the standing hypothesis that $\{(\mathbf{f}(x, w),-h(x, w)):|w| \leq 1\}$ is a bounded and uniformly convex subset of $\mathbb{R}^{n+1}$. So for each $p \in \mathbb{R}^{n}$, there exists a unique pair $(\mathbf{f}(x, w),-h(x, w))$ that maximizes the expression $\langle\mathbf{f}(x, w), p\rangle-h(x, w)$.

Now suppose that $u^{\epsilon} \rightarrow u$ uniformly, as $\epsilon \rightarrow 0$. It turns out that then the function $u$ is a viscosity solution of the PDE

$$
-f^{i}(x, \hat{w}) f^{j}(x, \hat{w}) u_{x_{i} x_{j}}=0 \text { in } U,
$$

for each $\hat{w} \in \operatorname{argmax}\{\langle\mathbf{f}(x, w), D u\rangle-h(x, w):|w| \leq 1\}$.

We can interpret (5.4) as a generalization of the $\infty$-Laplacian.

Aronsson's equation, forward and backwards Hamilton-Jacobi flows. Another generalization of the $\infty$-Laplacian is Aronsson's operator

$$
A_{H}[u]:=H_{p_{j}}(D u) H_{p_{i}}(D u) u_{x_{i} x_{j}}
$$

defined for $H: \mathbb{R}^{n} \rightarrow \mathbb{R}, H=H(p)$.

We sketch a situation where the Aronsson operator arises. Given $H$ as above, consider the pair of Hamilton-Jacobi equations

$$
\left\{\begin{aligned}
v_{t}+H(D v) & =0 & & (t>0) \\
v & =g & & (t=0)
\end{aligned}\right.
$$

and

$$
\left\{\begin{array}{rr}
w_{t}-H(D w)=0 & (t>0) \\
w=g & (t=0)
\end{array}\right.
$$


A degenerate nonlinear wave equation. We formally differentiate (5.6) with respect to $t$ and $x_{k}$, to find

$$
\begin{gathered}
v_{t t}+H_{p_{k}} v_{x_{k} t}=0, \\
v_{x_{k} t}+H_{p_{j}} v_{x_{k} x_{j}}=0
\end{gathered}
$$

for $k=1, \ldots, n$. Substitute the second equation into the first, to discover that

$$
v_{t t}-A_{H}[v]=0,
$$

for the Aronsson operator (5.5). Similarly, we deduce that

$$
w_{t t}-A_{H}[w]=0 .
$$

So the solutions $v, w$ of the "forward" and "backwards" Hamilton-Jacobi equations (5.6) and (5.7) formally solve the same nonlinear wave equation; a deduction that is certainly in general false, since $v$ and $w$ are not usually smooth. But we do have the rigorous conclusions that

$$
\begin{aligned}
& v=g-t H(D g)+\frac{t^{2}}{2} A_{H}[g]+o\left(t^{2}\right) \\
& w=g+t H(D g)+\frac{t^{2}}{2} A_{H}[g]+o\left(t^{2}\right)
\end{aligned}
$$

as $t \rightarrow 0$, valid for smooth initial data $g$. And we can average these expansions equations to knock out the $O(t)$ terms:

$$
\frac{v+w}{2}=g+\frac{t^{2}}{2} A_{H}[g]+o\left(t^{2}\right)
$$

A nonlinear parabolic PDE. To be more precise, we now introduce nonlinear semigroup notation, writing $v:=R(t) g$ to denote the unique viscosity solution of (5.6), and $w:=S(t) g$ to denote the unique viscosity solution of (5.7). Next, set

$$
F(t):=\frac{R(\sqrt{t})+S(\sqrt{t})}{2} \quad(t \geq 0)
$$

to record an average of the two dynamics, over the long time scale $\sqrt{t}$.

What happens if we repeatedly apply the nonlinear operator $F$ over shorter and shorter time intervals?? In $[\mathbf{B}-\mathbf{E}-\mathbf{J}]$ we demonstrate that the limit

$$
u:=\lim _{n \rightarrow \infty} F(t / n)^{n} g \quad(t>0)
$$

exists uniformly for each time $t \geq 0$; and $u$ is the unique viscosity solution of the parabolic equation

$$
\left\{\begin{aligned}
u_{t}-\frac{1}{2} A_{H}[u] & =0 & & (t>0) \\
u & =g & & (t=0) .
\end{aligned}\right.
$$

The proof uses one of my favorite tools of nonlinear analysis, the "Chernoff formula" for nonlinear semigroups, due to Brezis and Pazy $[\mathbf{B}-\mathbf{P}]$. 


\section{References}

[A1] G. Aronsson, Extension of functions satisfying Lipschitz conditions. Ark. Mat. 6 (1967) 551-561.

[A2] G. Aronsson, On the partial differential equation $u_{x}{ }^{2} u_{x x}+2 u_{x} u_{y} u_{x y}+u_{y}{ }^{2} u_{y y}=0$. Ark. Mat. 7 (1968), 395-425.

[A-C-J] G. Aronsson, M. Crandall, and P. Juutinen, A tour of the theory of absolutely minimizing functions, Bull. Amer. Math. Soc. 41 (2004), 439-505.

[B-E-J] E. N. Barron, L. C. Evans and R. Jensen, The infinity Laplacian, Aronsson's equation and their generalizations, paper to appear.

[B-P] H. Brezis and A. Pazy, Convergence and approximation of semigroups of nonlinear operators in Banach spaces, J. Functional Analysis 9 (1972), 63-74.

[C-E-G] M.Crandall, L. C. Evans and R. Gariepy, Optimal Lipschitz extensions and the infinity Laplacian, Calculus of Variations and Partial Differential Equations 13 (2001), 123139.

[C-E] M.Crandall and L. C. Evans, A remark on infinity harmonic functions, Electronic Journal of Differential Equations, Conf. 06, (2001), 123-129.

[E1] L. C. Evans, A new proof of local $C^{1, \alpha}$ regularity of solutions of certain degenerate partial differential equations, Journal of Differential Equations 45 (1982), 356-373.

[E2] L. C. Evans, Some min-max methods for the Hamilton-Jacobi equation, Indiana U Math J 33 (1984), 31-50.

[E3] L. C. Evans, Estimates for smooth absolutely minimizing Lipschitz extensions, Electronic Journal of Differential Equations 1 (1993).

[E-So] L. C. Evans and P. E. Souganidis, Differential games and representation formulas for solutions of Hamilton-Jacobi equations, Indiana University Mathematics Journal 33 (1984), 773-797.

[E-Sp] L. C. Evans and J. Spruck, Motion of level sets by mean curvature I, Journal of Differential Geometry 33 (1991), 635-681.

$[\mathrm{J}] \quad$ R. Jensen, Uniqueness of Lipschitz extensions: minimizing the sup norm of the gradient, Arch. Rational Mech. Analysis 123 (1993), 51-74.

[K-S] R. Kohn and S. Serfaty, A deterministic control-based approach to motion by mean curvature, to appear in Communications Pure and Applied Math

[LG] E. Le Gruyer, On absolutely minimizing Lipschitz extensions and the PDE $\Delta_{\infty}(u)=0$, preprint, 2004

[O] A. Oberman, Convergent difference schemes for the infinity Laplacian: construction of absolutely minimizing Lipschitz extensions, preprint

[P-S] Y. Peres and S. Sheffield, Tug of war with noise: a game theoretic view of the $p$ Laplacian, preprint, 2006.

[P-S-S-W] Y. Peres, O. Schramm, S. Sheffield and D. Wilson, Tug-of-war and the infinity Laplacian, preprint, 2005.

[S] O. Savin, $C^{1}$ regularity for infinity harmonic functions in two dimensions, Arch. Rational Mech. Analysis 176 (2005), 351-361.

Department of Mathematics, University of California, Berkeley, CA 94720

E-mail address: evans@math.berkeley.edu 